\title{
Two types of Poisson pencils and related quantum objects
}

\author{
D. Gurevich \\ ISTV, Université de Valenciennes, 59304 Valenciennes, France \\ J.Donin \\ Departement of Mathematics, Bar-Ilan University, 52900 \\ Ramat-Gan,Israel \\ V.Rubtsov \\ ITEP, Bol.Tcheremushkinskaya 25, 117259 Moscow, Russia
}

Dedicated to Alain Guichardet with regards and friendship.

\begin{abstract}
Two types of Poisson pencils connected to classical R-matrices and their quantum counterparts are considered. A representation theory of the quantum algebras related to some symmetric orbits in $\operatorname{sl}(n)^{*}$ is constructed. A twisted version of quantum mechanics is discussed.

Mathematics Subject Classification (1991): 17B37, 81R50.

Keywords: Poisson bracket, Poisson pencil, quantum group, quantum algebra, associative pencil, symmetric orbit, twisted quantum mechanics.
\end{abstract}

\section{Introduction}

There are (at least) two reasons for Poisson pencils (P.p.) to be currently of great interest. First, they play a very important role in the theory of 
integrable systems (in the so-called Magri-Lenart scheme). Second, they appear as infinitesimal (quasi-classical) objects in the construction of certain quantum homogeneous spaces. Roughly speaking, we can say that the P.p. arising in the framework of the latter construction are of the first type and those connected to integrable systems belong to the second type (while the integrable systems themselves are disregarded).

The main characteristic that joins together these two classes of P.p. is that a classical R-matrix participates in their construction. Let us recall that by a classical R-matrix on a simple Lie algebra $g$ one means a skew-symmetric $\left(R \in \wedge^{2}(g)\right)$ solution of the classical Yang-Baxter equation

$$
\left[R^{12}, R^{13}\right]+\left[R^{12}, R^{23}\right]+\left[R^{13}, R^{23}\right]=a \varphi, a \in k,
$$

where $\varphi$ is a unique (up to a factor) ad-invariant element belonging to $\wedge^{3}(g)$ ). In what follows we deal with the "canonical" classical R-matrix

$$
R=\frac{1}{2} \sum X_{\alpha} \wedge X_{-\alpha}
$$

where $\left\{H_{\alpha}, X_{\alpha}, X_{-\alpha}\right\}$ is a Cartan-Weyl-Chevalley base of a simple Lie algebra $g$ over the field $k=\mathbf{C}$. We assume that a triangular decomposition of the Lie algebra $g$ is fixed. The field $k=\mathbf{R}$ is also admitted, but in this case we consider the normal real forms of the Lie algebras corresponding to this triangular decomposition.

Thus, the R-matrix (1) enters the constructions of both type of P.p. under consideration. However, the constructions and properties of these types are completely different. Moreover, the methods of quantizing these two types of P.p. are completely different, as well as the properties of the resulting associative algebras. We will use the term associative pencils (a.p.) of the first (second) type for the families of these quantum algebras arising from the first (second) type P.p.

The ground object of the second type P.p. is a quadratic Poisson bracket (P.b.) of Sklyanin type. By this type we mean either the famous Sklyanin bracket円 or its various analogues: the elliptic Sklyanin algebras in the sense of

\footnotetext{
${ }^{1}$ Let us specify that this bracket is given by

$$
\{,\}_{S}=\{,\}_{l}-\{,\}_{r},\{f, g\}_{\epsilon}=\mu\left\langle\rho_{\epsilon}^{\otimes 2}(R), d f \otimes d g\right\rangle, \epsilon=l, r,
$$

where $\rho_{l}\left(\rho_{r}\right): g \rightarrow \operatorname{Vect}(\operatorname{Mat}(n))$ is a representation of the Lie algebra $g$ into the space of
} 
[S], the so-called second Gelfand-Dikii structures, etc. (Although we restrict ourselves to finite-dimensional Poisson varieties, we would like to note it is not reasonable to consider these structures as the infinite-dimensional analogues of P.p. on symmetric orbits in $g^{*}$, as it was suggested in [KRR]. The principal aim of the present paper is to make the difference between the two classes of P.p. related to classical R-matrices more transparent.)

All these P.b. (further denoted by $\{,\}_{2}$ ) are quadratic and their linearization gives rise to another (linear) P.b. (denoted by $\{,\}_{1}$ ) compatible with the initial one. As a result we have a P.p.

$$
\{,\}_{a, b}=a\{,\}_{1}+b\{,\}_{2}
$$

generated by these two brackets.

Now let us describe the corresponding quantum objects. We construct them in two steps. First, we quantize the Sklyanin bracket and get the well-known quadratic "RTT=TTR" algebra. Then by linearizing the determining quadratic relations (or more precisely, by applying a shift operator to the algebra, cf. below) we get a (second type) a.p. which is the quantum counterpart of the whole P.p. These second type P.p. and their quantum counterparts are considered in Section 2.

Let us note that the shift operator mentioned above does not give rise to any meaningful deformation and it is reduced to a mere change of base.

We are interested in P.p. and their quantum counterparts connected to symmetric orbits in $g^{*}$, where $g$ is a simple Lie algebra. It should be pointed out that the latter P.p. are not any reduction of the second type P.p. Let us say a little bit more about the origin of these P.p. on symmetric orbits.

It is well known that any orbit $\mathcal{O}_{x}$ of any semisimple element in $g^{*}$ can be equipped with the reduced Sklyanin (sometimes called Sklyanin-Drinfeld) bracket. One usually considers the reduction procedure for the compact forms of simple Lie algebras, but it is also valid for normal ones (the R-matrix for compact forms differs from (1) by the factor $\sqrt{-1}$ ).

It is worth noting that the symmetric orbits in $g^{*}$ admit a complementary nice property: the reduced Sklyanin bracket is compatible with the Kirillov-

right (left) invariant vector fields on $\operatorname{Mat}(n), R$ is the classical R-matrix (1) corresponding to $\operatorname{sl}(n)$ and $\langle$,$\rangle is the pairing between vector fields and differentials extended to the$ their tensor powers. Hereafter $\mu$ is the multiplication in the algebra under consideration. (Let us note that for other simple Lie groups $G$ analogous P.b. being extended to $\operatorname{Mat}(n)$ are no longer Poisson.) 
Kostant-Souriau (KKS) bracket. This fact was shown in [KRR], [DG2] (in [KRR] it was also shown that only symmetric orbits possess this property).

Moreover, as shown in [DG2], both components $\{,\}_{\epsilon}, \epsilon=l, r$ of the Sklyanin bracket (cf. footnote 1) become Poisson after being reduced to $\mathcal{O}_{x}$ and one of them (say, $\{,\}_{r}$ coincides up to factor with the KKS one if we identify $\mathcal{O}_{x}$ with right coset $\left.G / H\right)$.

Since the brackets $\{,\}_{\epsilon}, \epsilon=l, r$ are always compatible, we have a (first type) P.p. (2) on a symmetric orbit $\mathcal{O}_{x} \in g^{*}$ with $\{,\}_{1}=\{,\}_{l}^{\text {red }}$ and $\{,\}_{2}=\{,\}_{r}^{\text {red }}$ ("red" means reduced on $\left.\mathcal{O}_{x}\right)$ ).

The difference between these two types of P.p. also manifests itself on the quantum level. One usually represents quantum analogues of the Sklyanin bracket reduced to a semisimple orbit in terms of "quantum reduction" by means of pairs of quantum groups or their (restricted) dual objects. However, in this way it is not possible to represent the whole a.p. arising from the first type P.p. under consideration. We discuss here another, more explicit, way to describe the corresponding quantum a.p. Namely, we represent them as certain quotient algebras.

In virtue of the results of the paper [DS1], a formal quantization of the P.p. under consideration exists on any symmetric orbit. However, it is not so easy to describe the quantum objects explicitly or, in other words, to find systems of equations defining them. We consider here two ways to look for such a system in the case when the orbit $\mathcal{O}_{x}$ is a rank 1 symmetric space in $\operatorname{sl}(n)^{*}$, i.e., that of $S L(n) / S(L(n-1) \times L(1))$ type.

The first way was suggested in [DG1] (but there the system of equations was given in an inconsistent form). It uses the fact that such an orbit can be described by a system of quadratic equations (cf. Section 3). The second way consists in an attempt to construct a representation theory of the first type quantum algebras (cf. Section 4) and to compute the desired relations in the modules over these algebras. This approach is valid in principle for any symmetric orbit in $g^{*}$ for any simple Lie algebra $g$. This enables us to treat this

\footnotetext{
${ }^{2}$ Let us note that similar (first type) P.p. exists on certain nilpotent orbits in $g^{*}$. These pencils are generated by the KKS bracket and the so-called R-matrix bracket defined by

$$
\{,\}_{R}=\mu\left\langle\rho^{\otimes 2}(R), d f \otimes d g\right\rangle, \rho=\mathrm{ad}^{*} .
$$

In fact the R-matrix bracket is just that $\{,\}_{l}^{\text {red }}$ for any such orbit but only for the symmetric ones the brackets KKS and $\{,\}_{r}^{\text {red }}$ are proportional to each other. The reader is referred to $\mathrm{GP}$ where all orbits in $g^{*}$ possessing the above P.p. are classified.
} 
type of quantum algebras from the point of view of twisted quantum mechanics (Section 5). This means that quantum algebras and their representations are objects of a twisted (braided) category. (We consider the term "twisted" as more general, keeping the term "braided" for nonsymmetric categories).

Completing the Introduction, we would like to make two remarks. First, let us note that there is a number of papers devoted to q-analogues of special functions of mathematical physics. In fact they deal with quantum analogues of double cosets. Meanwhile, the problem of finding an explicit description of quantum symmetric spaces (in particular, orbits in $g^{*}$ ) is disregarded (in some sense the latter problem is more complicated). If the usual q-special functions arise from a one-parameter deformation of the classical objects, our approach enables us to consider their analogues arising from the twoparameter deformation. This approach will be developed in a joint paper of one of the author (D.G.) and L.Vainerman.

Second, we would like to emphasize that we consider the present paper as an intermediate review of the papers [DG1], [DGR], [G2] where the present topic (i.e., the problem of the explicit description of a.p. arising from quantization of certain P.p. associated with the "canonical" classical R-matrices) was discussed. Meanwhile, this topic is still in progress.

In what follows $U_{q}(g)$-Mod stands for the category of all finite-dimensional $U_{q}(g)$-modules and their inductive limits. The parameter $q$ is assumed to be generic (or formal, when we speak about flatness of deformation, in fact we do not distinguish these two meanings).

We dedicate this paper to our friend, professor Alain Guichardet. We acknowledge his warm hospitality at Ecole Polytechnique for a long time and benefited a lot from our numerous discussions and conversations.

This paper was finished during the visit of V.R. to the University Lille-1. He is thankful to his colleagues from the group of Mathematical Physics for their hospitality. The work of V.R. was supported partially by the grants RFFI-O1-01011, INTAS-93-2494 and INTAS-1010-CT93-0023. 


\section{Second type Poisson and associative pen- cils}

Let us fix a simple algebra $g=\operatorname{sl}(n), n \geq 2$ and consider the corresponding quadratic Skyanin bracket extended on the space Fun(Mat $(n))$ which is the algebra of polynomials on the matrix elements $a_{i}^{j}$. More precisely, this bracket is determined by the following multiplication table

$$
\begin{gathered}
\left\{a_{k}^{i}, a_{k}^{j}\right\}_{2}=a_{k}^{i} a_{k}^{j},\left\{a_{i}^{k}, a_{j}^{k}\right\}_{2}=a_{i}^{k} a_{j}^{k}, i<j ; \\
\left\{a_{i}^{l}, a_{k}^{j}\right\}_{2}=0, \quad\left\{a_{i}^{j}, a_{k}^{l}\right\}_{2}=2 a_{i}^{l} a_{k}^{j}, i<k, j<l .
\end{gathered}
$$

Let us linearize the bracket $\{,\}_{2}$. To do this we introduce a shift operator $\mathrm{Sh}_{h}$ which sends $a_{i}^{j}$ to $a_{i}^{j}+h \delta_{i}^{j}$ (we extend this operator onto the whole algebra Fun(Mat $(n))$ by multiplicativity). Then applying this operator to r.h.s. of the above formulas and taking the linear terms in $h$, we get a linear bracket with by the following multiplication table

$$
\begin{gathered}
\left\{a_{k}^{i}, a_{k}^{j}\right\}_{1}=\delta_{k}^{i} a_{k}^{j}+a_{k}^{i} \delta_{k}^{j},\left\{a_{i}^{k}, a_{j}^{k}\right\}_{1}=\delta_{i}^{k} a_{j}^{k}+a_{i}^{k} \delta_{j}^{k}, i<j \\
\left\{a_{i}^{l}, a_{k}^{j}\right\}_{1}=0,\left\{a_{i}^{j}, a_{k}^{l}\right\}_{1}=2\left(\delta_{i}^{l} a_{k}^{j}+a_{i}^{l} \delta_{k}^{j}\right), i<k, j<l .
\end{gathered}
$$

Proposition 1 The brackets $\{,\}_{1,2}$ are compatible.

Proof. This follows immediately from the following fact: the r.h.s. of the formulas for the bracket $\{,\}_{2}$ does not contain any summand of the form $a_{i}^{i} a_{j}^{j}$. This implies that the images of r.h.s. elements under the operator $\mathrm{Sh}_{h}$ do not contain any term quadratic in $h$. The details are left to the reader.

Remark 1 It is often more convenient to take, instead of $\left\{a_{i}^{j}\right\}$, the base consisting of $a_{i}^{j}, i \neq j, a_{1}^{1}-a_{i}^{i}, i>1$ and $a_{0}=\sum a_{i}^{i}$. Then the mentioned property can be reformulated as follows: the r.h.s. of the above formulas do not contain the term $\left(a_{0}\right)^{2}$ (with respect to these new generators the operator $\mathrm{Sh}_{h}$ acts nontrivially only on $\left.a_{0}\right)$. 
It is easy to see that the bracket $\{,\}_{1}$ can be represented in the form

$$
\left\{a_{i}^{j}, a_{k}^{l}\right\}_{1}=\left\{\mathbf{R}\left(a_{i}^{j}\right), a_{k}^{l}\right\}_{g l}+\left\{a_{i}^{j}, \mathbf{R}\left(a_{k}^{l}\right)\right\}_{g l} .
$$

Here $\{,\}_{g l}$ is the linear bracket corresponding to the Lie algebra $g l(n)$ (namely, $\left\{a_{i}^{j}, a_{k}^{l}\right\}_{g l}=a_{i}^{l} \delta_{k}^{j}-a_{k}^{j} \delta_{i}^{l}$ ) and $\mathbf{R}: W \rightarrow W$ is an operator defined in the space $W=\operatorname{Span}\left(a_{i}^{j}\right)$ as follows $\mathbf{R}\left(a_{i}^{j}\right)=\operatorname{sign}(j-i) a_{i}^{j}$ (we assume that $\operatorname{sign}(0)=0)$.

Thus, the space $W$ is equipped with two Lie algebra structures. The first one is $g l(n)$ and the second one corresponds to the Poisson bracket $\{,\}_{1}$ and therefore we have a double Lie algebra structure on the space $W$ according to the terminology of [S-T] (our construction coincides with [S-T] up to a factor).

Let us consider now the quantum analogue of the above P.p.

Let $U_{q}(g)$ be the Drinfeld-Jimbo quantum group corresponding to $g$ and $\mathcal{R}$ be the universal quantum R-matrix. Consider the linear space $V$ of vector fundamental representation $\rho: g \rightarrow \operatorname{End}(V)$ of the initial Lie algebra $g$. Denote by $\rho_{q}$ the corresponding representation of the quantum group $U_{q}(g)$ to $\operatorname{End}(V)$ (we assume that $\rho_{q} \rightarrow \rho$ when $q \rightarrow 1$ ). Then the operator $S: V^{\otimes 2} \rightarrow$ $V^{\otimes 2}$ defined as follows $S=\sigma \rho^{\otimes 2}(\mathcal{R})$ where $\sigma$ is the flip $(\sigma(x \otimes y)=y \otimes x)$ satisfies the quantum Yang-Baxter equation (QYBE)

$$
S^{12} S^{23} S^{12}=S^{23} S^{12} S^{23}, S^{12}=S \otimes \mathrm{id}, S^{23}=\mathrm{id} \otimes S
$$

and possesses two eigenvalues (we recall that $g=\operatorname{sl}(n)$ ).

Let us identify now the space $W$ with $V \otimes V^{*}$ and equip it with the operator $S_{W}=S \otimes\left(S^{*}\right)^{-1}: W^{\otimes 2} \rightarrow W^{\otimes 2}$, where $S^{*}$ is defined with respect to the pairing

$$
\langle x \otimes y, a \otimes b\rangle=\langle x, a\rangle\langle y, b\rangle, x, y \in V, a, b \in V^{*} .
$$

Let us fix a base $\left\{a_{i}\right\} \in V$. Let $\left\{a^{i}\right\} \in V^{*}$ be the dual base and set $a_{i}^{j}=a_{i} \otimes a^{j}$. Then we have the following explicit form for the operator $S_{W}$ :

$$
S_{W}\left(a_{i}^{k} \otimes a_{j}^{l}\right)=S_{i j}^{m n} S_{p q}^{-1 k l}\left(a_{m}^{p} \otimes a_{n}^{q}\right) \text { where } S\left(a_{i} \otimes a_{j}\right)=S_{i j}^{k l} a_{k} \otimes a_{l} .
$$

It is easy to see that the operator $S_{W}$ also satisfies the QYBE and possesses 1 as an eigenvalue. Then it is natural to introduce deformed analogues of symmetric and skew-symmetric subspaces of the space $W^{\otimes 2}$ as follows

$$
I_{-}^{q}=\operatorname{Im}\left(S_{W}-\mathrm{id}\right), I_{+}^{q}=\operatorname{Ker}\left(S_{W}-\mathrm{id}\right) .
$$


Let us describe explicitly the operator $S$ and the spaces $I_{ \pm}$:

$$
\begin{gathered}
S\left(a_{i} \otimes a_{j}\right)=(q-1) \delta_{i, j} a_{i} \otimes a_{j}+a_{j} \otimes a_{i}+\sum_{i<j}\left(q-q^{-1}\right) a_{i} \otimes a_{j}, \\
I_{-}^{q}=\operatorname{Span}\left(a_{k}^{i} a_{k}^{j}-q a_{k}^{j} a_{k}^{i}, a_{i}^{k} a_{j}^{k}-q a_{j}^{k} a_{i}^{k}, i<j ;\right. \\
\left.a_{i}^{l} a_{k}^{j}-a_{k}^{j} a_{i}^{l}, a_{i}^{j} a_{k}^{l}-a_{k}^{l} a_{i}^{j}-\left(q-q^{-1}\right) a_{k}^{j} a_{i}^{l}, i<k, j<l\right)
\end{gathered}
$$

and

$$
\begin{gathered}
I_{+}^{q}=\operatorname{Span}\left(\left(a_{i}^{k}\right)^{2}, q a_{k}^{i} a_{k}^{j}+a_{k}^{j} a_{k}^{i}, q a_{i}^{k} a_{j}^{k}+a_{j}^{k} a_{i}^{k}, i<j ;\right. \\
\left.a_{i}^{j} a_{k}^{l}+a_{k}^{l} a_{i}^{j}, a_{i}^{l} a_{k}^{j}+a_{k}^{j} a_{i}^{l}+\left(q-q^{-1}\right) a_{i}^{j} a_{k}^{l}, i<k, j<l\right) .
\end{gathered}
$$

Let us introduce also the quotient algebra $A_{0, q}=T(W) /\left\{I_{-}^{q}\right\}$. In what follows $T(W)$ denotes the free tensor algebra of the space $W$ and $\{I\}$ denotes the two-sided ideal generated by the set $I \subset T(W)$ (here $I=I_{-}^{q}$ is a subspace of the space $\left.W^{\otimes 2}\right)$.

It is well known (cf. [RTF]) that the quantum analogue $\operatorname{Fun}_{q}(S L(n))$ of the space $\operatorname{Fun}(S L(n))$ is the quotient algebra of $A_{0, q}$ over the ideal generated by the element $\operatorname{det}_{q}-1$ ( $\operatorname{det}_{q}$ is the so-called "quantum determinant"). Moreover, the latter quotient algebra can be endowed with a Hopf structure. However, we are interested rather in the algebra $A_{0, q}$ itself. This algebra is quadratic and is a flat deformation of the classical counterpart, namely, of the symmetric algebra $\operatorname{Sym}(W)$ of the space $W$ (cf. DS2 for details). Let us note that the skew-symmetric algebra of $W$ has also an evident quantum analogue $T(W) /\left\{I_{+}^{q}\right\}$.

Now we want to introduce two parameter deformation of the algebra $\operatorname{Sym}(W)$ quantizing the whole P.p. under consideration. This deformation can be realized by means of the same shift operator as above. Applying this operator to $I_{-}^{q}$ we obtain the following space

$$
\begin{gathered}
J_{h, q}=\operatorname{Span}\left(a_{k}^{i} a_{k}^{j}-q a_{k}^{j} a_{k}^{i}-h\left(\delta_{k}^{i} a_{k}^{j}+a_{k}^{i} \delta_{k}^{j}\right),\right. \\
a_{i}^{k} a_{j}^{k}-q a_{j}^{k} a_{i}^{k}-h\left(\delta_{i}^{k} a_{j}^{k}+a_{i}^{k} \delta_{j}^{k}\right), i<j ; a_{i}^{l} a_{k}^{j}-a_{k}^{j} a_{i}^{l}, \\
\left.a_{i}^{j} a_{k}^{l}-a_{k}^{l} a_{i}^{j}-\left(q-q^{-1}\right) a_{k}^{j} a_{i}^{l}-h m\left(\delta_{i}^{l} a_{k}^{j}+a_{i}^{l} \delta_{k}^{j}\right), i<k, j<l\right),
\end{gathered}
$$

where $m=1+q^{-1}$ (we have realized here a substitution $h(q-1) \rightarrow h$ ).

Let us introduce the algebra $A_{h, q}=T(W) /\left\{J_{h, q}\right\}$. It is evident that this algebra is a flat deformation of the initial commutative algebra $A_{0,1}$ since the 
passage from the algebra $A_{0, q}$ to that $A_{h, q}$ is trivial from the deformation point of view and reduces to a change of a base. Thus, we have constructed the a.p. $A_{h, q}$, which is a quantum analogue of the P.p. under consideration (it is easy to see that quasi-classical term of this two parameter deformation is just the above second type P.p.).

A similar method can be applied to quantize the P.p. generated by the elliptic Sklyanin P.b [S]. This bracket (denoted also by $\{,\}_{2}$ ) is determined by the following multiplication table

$$
\left\{S_{1} S_{0}\right\}=2 J_{23} S_{2} S_{3}, \quad\left\{S_{1} S_{2}\right\}=-2 S_{0} S_{1}
$$

and their cyclic permutations with respect to the indices $(1,2,3)$ with some elliptic functions $J_{i j}$ (cf. [S]). The linearization of this bracket defined by the shift operator $S_{0} \rightarrow S_{0}+h, S_{i} \rightarrow S_{i}, i \neq 0\left(S_{0}\right.$ plays here the role of the element $a_{0}$ from Remark 1$)$ gives rise to the linear one corresponding to the Lie algebra $g=s o(3) \oplus k$. The brackets are compatible (by the same reason as above).

To quantize the P.p. generated by them, it suffices to quantize the bracket $\{,\}_{2}$ and apply the shift operator. It is well known (cf. [S]) that a quantum analogue of the bracket $\{,\}_{2}$ is the quotient algebra $T(W) /\{I\}$ where $W=\operatorname{Span}\left(S_{0}, \ldots, S_{3}\right)$ and $\{I\}$ is two-sided ideal in $T(W)$ generated by the elements

$$
S_{1} S_{0}-S_{0} S_{1}+i J_{23}\left(S_{2} S_{3}+S_{3} S_{2}\right), S_{1} S_{2}-S_{2} S_{1}-i\left(S_{0} S_{3}+S_{3} S_{0}\right)
$$

and their cyclic permutations with some elliptic functions $J_{i j}$.

We leave to the reader the explicit description of the resulting two parameter quantum a.p.

The algebra $T(W) /\{I\}$ originally defined by E. Sklyanin is an elliptic analogue of the symmetric algebra of the space $W$. Unfortunately, we do not know any natural elliptic analogue of the skew-symmetric one (it seems very plausible that such analogue does not exist).

Remark 2 There are other quantum analogues of symmetric and skewsymmetric algebras of the space $W$ defined by the so-called reflection equations (RE)

$$
S u_{1} S u_{1}=u_{1} S u_{1} S, u_{1}=u \otimes 1, u=\left(u_{i}^{j}\right)
$$


where $S$ is a solution of (3). Conjecturally this "RE algebra" is a flat deformation of its classical counterpart (assuming $S$ to be of Hecke type). At least a necessary condition for flatness of a deformation, i.e., the existence of a P.b. as a quasiclassical term of the deformation, is satisfied. The mentioned bracket is also quadratic and admits a linearization. These two brackets are also compatible. We get a quantization of the P.p. generated by them by applying to the above algebra a shift operator (for details the reader is referred to [I], [IP], [G2]).

\section{Rank 1 quantum orbits}

In what follows we consider the P.p. and their quantum counterparts connected to symmetric orbits. These P.p. were described in the Introduction: they are generated by the KKS bracket and by the so-called R-matrix bracket.

We want to describe the corresponding quantum a.p. explicitly by means of a system of equations. In the present section we recall the method suggested in [DG1] to look for such systems. Unfortunately, it is valid only for rank 1 symmetric spaces, namely, those of $S L(n) / S(L(n-1) \times L(1))$ type.

Let us fix a Lie algebra $g=\operatorname{sl}(n)$ and an element $x=\omega \in h^{*}$, where $h \subset g=s l(n)$ is the Cartan subalgebra (a triangular decomposition of $g$ is assumed to be fixed). We consider $\omega$ as an element of $g^{*}$ extending it by zero to the nilpotent subalgebras. Let $\mathcal{O}_{\omega}$ be its orbit in $g^{*}$.

It is well known that this orbit is symmetric iff $\omega\left(h_{i}\right)=0$ for all $i \neq i_{0}$ where $\left\{h_{i}=e_{i, i}-e_{i+1, i+1}, i=0, \ldots, n-1\right\}$ is the standard basis in $h$. The rank of this symmetric space is $r k\left(\mathcal{O}_{\omega}\right)=\min \left(i_{0}, n-i_{0}\right)$. We assume here that $r k\left(\mathcal{O}_{\omega}\right)=1$. So we have $i_{0}=1$ or $i_{0}=n-1$. To the sake of concreteness we set $i_{0}=1$.

Thus, we have a family $\mathcal{O}_{\omega}$ of such orbits parametrized by the value $\omega\left(h_{1}\right) \in k$ ( $k=\mathbf{C}$ or $k=\mathbf{R}$ corresponding to the case under question). Let us represent the algebra $\operatorname{Fun}\left(\mathcal{O}_{\omega}\right)$ as a quotient algebra of $\operatorname{Sym}(g)=\operatorname{Fun}\left(g^{*}\right)$. It is not difficult to show that this algebra can be described by a system of equations quadratic in generators of the space $g$.

Let us describe this system explicitly. Consider the space $V=g$ as an object of the category $g$-Mod. Let $V^{\otimes 2}=\oplus V_{\beta}$ be a decomposition of $V^{\otimes 2}$ into a direct sum of isotypic $g$-modules, where $\beta$ denotes the highest weight (h.w.) of the corresponding module. Let $\alpha$ be h.w. of $g$ as a $g$-module. 
Then in the above decomposition there is a trivial module $V_{0}(\beta=0)$, a module $V_{2 \alpha}$ of the h.w. $\beta=2 \alpha$, and two irreducible modules isomorphic to $g$ itself. One of them belongs to the space $I_{+} \subset V^{\otimes 2}$ of symmetric tensors and the other one to the space $I_{-} \subset V^{\otimes 2}$ of skew-symmetric tensors. We will use the notation $V_{+}\left(V_{-}\right)$for the former (latter) of them.

Note that a similar decomposition is valid for other simple Lie algebras, but for them there exists only one module isomorphic to $g$. It belongs to the skew-symmetric part and we keep the notation $V_{-}$for it.

Let us denote by $C$ the generator (called Casimir) of the module $V_{0}$. Then the orbit $\mathcal{O}_{\omega}$ is given by the following system of equations

$$
I_{-}=0 ; V_{\beta}=0 \forall V_{\beta} \subset I_{+}, V_{\beta} \notin\left\{V_{0}, V_{+}, V_{2 \alpha}\right\} ; C=c_{0}, V_{+}=c_{1} g
$$

with some factors $c_{0}$ and $c_{1}$ (the latter relation is a symbolic way to write the system which arises if we choose some bases in $g$ and $V_{+}$and equate the highest weight element of $V_{+}$to that one of $g$ times a factor $c_{1}$ and consider all the relations that follow).

It is not difficult to find the values of the factors $c_{i}, i=0,1$. It suffices to substitute "the point" $\omega$ to this system. (Let us note that in DG1 this system was given in a inconsistent form, where the condition $c_{1}=0$ was assumed.)

A similar procedure can be realized in the category $U_{q}(g)$-Mod. More precisely, we equip the space $V$ with the structure of a $U_{q}(g)$-module deforming the initial $g$-module structure on $V$ (there exists a regular way to convert any finite-dimensional $g$-module into a $U_{q}(g)$ one, cf., i.e., [CP]). Using the comultiplication in the quantum algebra $U_{q}(g)$, we equip the space $V^{\otimes 2}$ with an $U_{q}(g)$-module structure and decompose it as above into a direct sum of irreducible $U_{q}(g)$-modules. We will denote these components by $V_{\beta}^{q}$. By $C_{q}$ we denote a generator of the module $V_{0}^{q}$ (we call $C_{q}$ a braided Casimir).

Then we can impose a similar system of equations by replacing all $V_{\beta}$ participating in the system (4) by their quantum (or q-) analogues $V_{\beta}^{q}$ and $C$ by $C_{q}$. The only problem is: what are the proper values of the factors $c_{i}(q), i=0,1$, which now depend on $q$.

In [DG1 the following way to find out a proper system of equations was suggested. Let us consider the data $\left(V, I^{q}, \nu_{0}, \nu_{1}\right)$, where $I^{q}=V^{\otimes 2} \backslash V_{2 \alpha}^{q}$ and $\nu_{i}, i=0,1$ are two $U_{q}(g)$-morphisms defined as follows $\nu_{0}: V_{0}^{q} \rightarrow k\left(\nu_{0}\left(C_{q}\right)=\right.$ $c_{0}$ ) and $\nu_{1}: V_{+}^{q} \rightarrow c_{1} g$ (all other components of $V^{\otimes 2} \backslash V_{2 \alpha}^{q}$ are sent by $\nu_{i}$ to zero). 
Let us also consider the graded quadratic algebra $T(V) /\left\{I^{q}\right\}$ and its filtered analogue $T(V) /\left\{I_{c_{0}, c_{1}}^{q}\right\}$, where $\left\{I_{c_{0}, c_{1}}^{q}\right\}$ the ideal generated by the elements

$$
I_{-}^{q}, V_{\beta} \subset I_{+}^{q}, V_{\beta} \notin\left\{V_{0}, V_{+}, V_{2 \alpha}\right\}, C-c_{0}(q), V_{+}^{q}-c_{1}(q) g
$$

(see the end of this Section for the definition of the spaces $I_{ \pm}^{q}$ ).

Let us note that the algebra $T(V) /\left\{I^{q}\right\}$ is the quantum analogue of the function space on the cone and it is a flat deformation of its classical counterpart. The flatness can be shown, e.g., in the way suggested in [DG2, where an intertwining operator of the initial commutative product and the deformed one is given. Moreover, the algebra $T(V) /\left\{I^{q}\right\}$ is Koszul for a generic $q$ since it is so for the case $q=1$ by virtue of $[\mathbb{B}]$.

Let us assume now that the above data satisfy the following system

$$
\begin{aligned}
& \operatorname{Im}\left(\nu_{1} \otimes \mathrm{id}-\mathrm{id} \otimes \nu_{1}\right)(I \otimes V \bigcap V \otimes I) \subset I, \\
& \left(\nu_{1}\left(\nu_{1} \otimes \mathrm{id}-\mathrm{id} \otimes \nu_{1}\right)+\nu_{0} \otimes \mathrm{id}-\mathrm{id} \otimes \nu_{0}\right)(I \otimes V \bigcap V \otimes I)=0, \\
& \nu_{0}\left(\nu_{1} \otimes \mathrm{id}-\mathrm{id} \otimes \nu_{1}\right)(I \otimes V \bigcap V \otimes I)=0 .
\end{aligned}
$$

Then by virtue of the PBW theorem in the form of [BG] we can conclude that the algebra $T(V) /\left\{I_{c_{0}, c_{1}}^{q}\right\}$ is a flat deformation of the algebra $T(V) /\left\{I^{q}\right\}$.

Let us note that the above conditions represent a more general form of the Jacobi relation connected to deformation theory. Thus, if the above form of the Jacobi identity is fulfilled, the algebra $T(V) /\left\{I_{c_{0}, c_{1}}^{q}\right\}$ is a flat deformation of the orbit $\mathcal{O}_{\omega}$ (more precisely, of the function algebra on it).

So, the proper quantities $c_{i}(q), i=0,1$, if they exist, can be found from the above equations. However, a priori it is not clear why such quantities exist. Let us assume here that they exist and denote by $A_{0, q}$ the corresponding algebra $T(V) /\left\{I_{c_{0}, c_{1}}^{q}\right\}$. We are interested in its further deformation.

To do this, we begin by discussing the following question: what is the deformational quantization of the KKS bracket? We want to represent the latter quantum object also as a quotient of the enveloping algebra $U(\operatorname{sl}(n))_{h}$ The index $h$ here means that we have introduced a factor $h$ in the bracket in the definition of the enveloping algebra, i.e.,

$$
U(g)_{h}=T(g) /\{x y-y x-h[x, y]\} .
$$

There are some factors $c_{i}(h), i=0,1$, now depending on $h$ such that the quotient algebra $A_{h, 0}=U(\operatorname{sl}(n))_{h} /\{J\}$, where the ideal $\{J\}$ is generated 
by the family of elements from (4) lying in $I_{+}$but with new $c_{i}(h)$, is a flat deformation of the initial algebra corresponding to the case $h=0$. These factors can be also found by means of the above form of Jacobi identity.

This approach can also be applied in order to get the quantum algebras corresponding to the whole P.p. under consideration, since these algebras are quadratic as well. The only problem is: what is the proper quantum analogue of the algebra $U(\operatorname{sl}(n))_{h}$ ? Or, in other words, what is a consistent way to introduce a quantum analogue of the Lie bracket?

We will introduce a q-generalization of the ordinary Lie bracket by means of the following

Definition 1 Let $g$ be a simple Lie algebra equipped with a $U_{q}(g)$-module structure and $V_{\beta}^{q}$ be the irreducible modules in the category $U_{q}(g)$-Mod entering its tensor square. We call $q$-Lie bracket the operator $[,]_{q}: V^{\otimes 2} \rightarrow V$ defined as follows

$$
\left.[,]_{q}\right|_{V_{\beta}^{q}}=0 \text { for all } V_{\beta}^{q} \neq V_{-}^{q}
$$

and $[,]_{q}: V_{-}^{q} \rightarrow V$ is a $U_{q}(g)$-isomorphism.

Let us observe that in this way the q-Lie bracket is defined up to a factor. We fix this factor and introduce the q-analogue of the algebra $U(g)_{h}$ as follows

$$
U(g)_{h, q}=T(V) /\left\{\operatorname{Im}\left(\mathrm{id}-h[,]_{q}\right) I_{-}^{q}\right\} .
$$

The space $V=g$ equipped with this bracket will be denoted by $\bar{g}$ and called

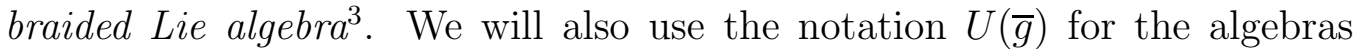
$U(g)_{1, q}$. Let us note that this is another, as compared with the quantum group $U_{q}(g)$, q-analogue of the enveloping algebra $U(g)$, but the deformation $U(g) \rightarrow U(g)_{h, q}$ is not flat except for the $s l(2)$ case. However, some quotient algebras of this algebra are flat deformations of their classical counterparts.

Let us return to the case $g=\operatorname{sl}(n)$ and introduce the first type a.p. $A_{h, q}$ as the quotient algebra of $U(\operatorname{sl}(n))_{h, q}$ by the ideal

$$
\left\{C_{q}-c_{0}, V_{+}^{q}-c_{1} V, I_{+}^{q} \backslash\left(V_{2 \alpha}^{q} \oplus V_{0}^{q} \oplus V_{+}^{q}\right)\right\}
$$

with some factors $c_{i}(h, q), i=0,1$.

\footnotetext{
${ }^{3}$ Other definitions of q-counterparts of the Lie algebras have been suggested recently in [?] and [?]. It is very plausible that they are equivalent to ours.
} 
In order to look for the consistent factors $c_{i}(h, q)$, we must only modify the above morphism $\nu_{1}$ on the "q-skew-symmetric" subspace $I_{-}^{q}$ by setting $\nu_{1}: V_{-}^{q} \rightarrow h g$ (all other components are still sent by $\nu_{1}$ to zero) and verify the above form of Jacobi identity. This provides us with the factors $c_{i}(h, q)$ ensuring the flatness of the deformation of the function algebra on the orbit $\mathcal{O}_{\omega}$ under consideration.

Let us emphasize that the existence of the proper factors $c_{i}(h, q)$, as well as that of the above factors $c_{i}(q)$, can be deduced from the paper [DS1], where a formal quantization of the P.p. under question was considered. In the next Section we discuss another way to look for appropriate factors $c_{i}(h, q)$.

It remains only to note that the quasiclassical term of two-parameter deformation $\operatorname{Fun}\left(\mathcal{O}_{\omega}\right) \rightarrow A_{h, q}$ is just the above P.p. on the orbit $\mathcal{O}_{\omega}$ (cf. [DG1]).

It is worth to note that the product in the resulting quantum algebras $A_{h, q}$ is $U_{q}(s l(n))$-invariant. This means that the following property

$$
X \mu(a \otimes b)=\mu\left(X_{1}(a) \otimes X_{2}(b)\right), a, b \in A_{h, q}, X \in U_{q}(\operatorname{sl}(n))
$$

is satisfied. (Hereafter we use Sweedler's notation $X_{1} \otimes X_{2}$ for $\Delta(X)$.) This property follows immediately from the construction of the algebras. This algebra is the inductive limit of finite-dimensional $U_{q}(s l(n))$-modules (moreover, it is multiplicity free, i.e., the multiplicity of each irreducible $U_{q}(g)$ module is $\leq 1)$. Thus, this algebra belongs to the category $U_{q}(\operatorname{sl}(n))$-Mod.

Let us note that a particular case of the a.p. $A_{h, q}$ is the algebra $A_{0, q}$ arising as the result of quantization of the only R-matrix bracket. This algebra is commutative in the category $U_{q}(s l(n))$-Mod in the following sense. This category is balanced (see $[\overline{C P}$ for the definition). Moreover, for any two finite-dimensional objects $U$ and $V$ of this category there exists an involutive operator $\widetilde{S}: U \otimes V \rightarrow V \otimes U$ such that it is a $U_{q}(g)$-morphism, it commutes with $S$ and it is a deformation of the flip.

Then $I_{ \pm}^{q}=\operatorname{Im}(\mathrm{id} \pm \widetilde{S})$ with $\widetilde{S}: V^{\otimes 2} \rightarrow V^{\otimes 2}$. Using the fact that the algebra $A_{h, q}$ can be decomposed into a direct sum of finite-dimensional objects of the category $U_{q}(\operatorname{sl}(n))$-Mod we can extend the operator $\widetilde{S}$ onto $A_{h, q}^{\otimes 2}$. Then the above mentioned commutativity of the algebra $A_{0, q}$ means that the multiplication operator $\mu$ satisfies the following relation $\mu=\mu \widetilde{S}$. A proof of this fact can be obtained from DS1. Strictly speaking, just the algebra $A_{0, q}$ is the quantum analogue of the orbit under consideration. 
Let us note that the above method can be also applied to find the equations describing quantum algebras arising from similar P.p. on certain nilpotent orbits in $g^{*}$ (see the Introduction). Thus, such a.p. related to the highest weight element orbits in $g^{*}$ can be defined by means of the above ideal $I_{c_{0}, c_{1}}^{q}$, but with $c_{0}(h, q)=0$ and with a suitable $c_{1}(h, q)$.

\section{Modules for first type quantum algebras}

In the previous section we discussed the first step of quantization procedure. The resulting object of this step is an a.p. represented as a quotient algebras over some suitable ideal. Now we want to consider the second step of quantization, consisting in an attempt to represent the above algebras in certain linear spaces. At this step the difference between two types of quantum algebras (a.p.) under question becomes clearer.

Moreover, this step provides us with another way to look for consistent factors $c_{i}(h, q)$. Briefly speaking, this method reduces to the computation of the factor $c_{i}(h, q)$ on the image of the first type algebras into the space $\operatorname{End}(V)$.

To do this, we recall a natural way to equip the space of endomorphisms of a $U_{q}(g)$-module with a $U_{q}(g)$-module structure. Let $U^{q}$ be a (finite-dimensional) $U_{q}(g)$-module and $\rho_{q}: U_{q}(g) \rightarrow \operatorname{End}\left(U^{q}\right)$ be the corresponding representation. Let us introduce the representation $\rho_{q}^{\text {End }}: U_{q}(g) \rightarrow$ $\operatorname{End}\left(\operatorname{End}\left(U^{q}\right)\right)$ by putting

$$
\rho_{q}^{\text {End }}(a) M=\rho\left(a_{1}\right) \circ M \circ \rho\left(\gamma\left(a_{2}\right)\right), a \in U_{q}(g), M \in \operatorname{End}\left(U^{q}\right) .
$$

We denote the matrix product by o, while $\gamma$ is the antipode in $U_{q}(g)$.

We deal with a coordinate representation of module elements. We consider the endomorphisms as matrices and their action is the left multiplication by these matrices.

Let us note that this way of equipping $\operatorname{End}\left(U^{q}\right)$ with a $U_{q}(g)$-module structure is compatible with the matrix product in it in the following sense:

$$
\rho_{q}^{\text {End }}(a)\left(M_{1} \circ M_{2}\right)=\rho_{q}^{\text {End }}\left(a_{1}\right) M_{1} \circ \rho_{q}^{\text {End }}\left(a_{2}\right) M_{2} .
$$

This means that $\circ: \operatorname{End}\left(U^{q}\right)^{\otimes 2} \rightarrow \operatorname{End}\left(U^{q}\right)$ is a $U_{q}(g)$-morphism.

Now we will introduce a useful notion for constructing a representation theory of the algebras under consideration. 
Definition 2 Let $\bar{g}$ be a braided Lie algebra. We say that a $U_{q}(g)$-module $U^{q}$ is a braided module or, more precisely, a braided $\bar{g}$-module if it can be equipped with a structure of a $U(\bar{g})$-module and the representation $\rho$ : $U(\bar{g}) \rightarrow \operatorname{End}\left(U^{q}\right)$ is a $U_{q}(g)$-morphism. We also say that the classical counterpart $U=U^{1}$ of the $U_{q}(g)$-module $U^{q}$ allows braiding.

A natural way to construct braided modules is given by the following

Proposition 2 Let $U^{q}$ be a $U_{q}(g)$-module. If the decomposition $\operatorname{End}\left(U^{q}\right)=$ $\oplus V_{\gamma}^{q}$ of the $U_{q}(g)$-module $\operatorname{End}\left(U^{q}\right)$ into the direct sum of irreducible $U_{q}(g)$ modules is such that

1. it does not contain modules isomorphic to $V_{\beta}^{q} \subset I_{-}^{q}$ apart from those isomorphic to $V^{q}$, where by $V^{q}$ we denote $g=V$ equipped with the $U_{q}(g)$ module structure,

2. the multiplicity of the module $V^{q}$ is 1 , then $U^{q}$ can be equipped with a braided module structure (briefly, braided structure).

Proof. By the assumption, there exists a unique $U_{q}(g)$-submodule in $\operatorname{End}\left(U^{q}\right)$, isomorphic to $V^{q}$. Consider a $U_{q}(g)$-morphism defined up to a factor

$$
\rho: V^{q} \rightarrow \operatorname{End}\left(U^{q}\right)
$$

This map is an almost representation of the braided Lie algebra $\bar{g}$ in the sense of the following

Definition 3 We say that a map (5) is an almost representation of the braided Lie algebra $\bar{g}$ if it is a $U_{q}(g)$-morphism and the following properties are satisfied

1. $\circ \rho^{\otimes 2}\left(V_{\beta}^{q}\right)=0$ for all $V_{\beta}^{q} \subset I_{-}^{q}$ apart from that $V_{-}^{q}$,

2. $\circ \rho^{\otimes 2} V_{-}^{q}=\nu \rho[,]_{q} V_{-}^{q}$ with some $\nu \neq 0$.

In a more explicit form these conditions can be reformulated as follows. If the elements $\left\{b_{k, \beta}^{i, j} u_{i} u_{j}, 1 \leq k \leq \operatorname{dim} V_{\beta}^{q}\right\}$ form a basis of the space $V_{\beta}^{q} \subset I_{-}^{q}$ and similarly the elements $\left\{b_{k,-}^{i, j} u_{i} u_{j}, 1 \leq k \leq \operatorname{dim} V_{-}^{q}\right\}$ form a basis of the space $V_{-}^{q}$ then

$$
b_{k, \beta}^{i, j} \rho\left(u_{i}\right) \rho\left(u_{j}\right)=0 \text { if } V_{\beta}^{q} \neq V_{-}^{q} \text { and } b_{k,-}^{i, j} \rho\left(u_{i}\right) \rho\left(u_{j}\right)=\nu b_{k,-}^{i, j} \rho\left(\left[u_{i}, u_{j}\right]_{q}\right) .
$$


Let us complete the proof. The image of the composed map $\circ \rho^{\otimes 2}\left(V_{-}^{q}\right)$ is isomorphic to the $U_{q}(g)$-module $V^{q}$ since $\rho$ and o are $U_{q}(g)$-morphisms. Such a module in the decomposition of $\operatorname{End}\left(U^{q}\right)$ is unique, therefore the image of the space $[,]_{q} V_{-}^{q}=V^{q}$ with respect to the morphism $\rho$ coincides with the previous one (it suffices to show that the images of the highest weight element of the module $V_{-}^{q}$ with respect to both operators coincide up to a factor). This gives the second property of Definition 2 (the property that $\nu \neq 0$ for a generic $q$ follows from the fact that this is so for $q=1$ ).

The first property of the Definition follows from the fact that $\operatorname{End}\left(U^{q}\right)$ does not contain any modules isomorphic to $V_{\beta}^{q} \subset I_{-}^{q}, V_{\beta}^{q} \neq V_{-}^{q}$. Finally, changing the scale, i.e., considering the map $\rho_{\nu}=\nu^{-1} \rho$ instead of $\rho$, we get a representation of the algebra $U(\bar{g})$. This completes the proof.

A natural question arises: how many $U_{q}(g)$-modules can be converted into braided ones or, in other words, how many $g$-modules allow braiding? The answer to this question for the $\operatorname{sl}(n)$-case is given by the following proposition, which can be proved by straightforward computations using Young diagram techniques.

Proposition 3 Let $\omega$ be a fundamental weight of the Lie algebra sl( $n)$. Then the $\operatorname{sl}(n)$-modules $V_{k \omega}$ (for any nonnegative integer $k$ ) allow braiding. In other words, their q-analogues $V_{k \omega}^{q}$ are braided modules.

For other simple Lie algebras, $g$ it seems very plausible that a similar statement is valid for fundamental weights $\omega$ such that their orbits in $g^{*}$ are symmetric (in the $s l(n)$-case all fundamental weights satisfy this condition). Note that all orbits of such type have been classified by E. Cartan (cf. [KRR].

Let us now discuss how the braided modules can be used to find the above factors $c_{i}(h, q)$.

Once more we set $g=\operatorname{sl}(n)$ and we take as fundamental the weight $\omega$ such that one $\omega\left(h_{1}\right)=1, \omega\left(h_{i}\right)=0, i>1$. Then we consider the $U(\operatorname{sl}(n))$ module $V_{k \omega}^{q}$ (which is in fact $V_{k \omega}$ equipped with a representation $\rho_{q}: U_{q}(g) \rightarrow$ $\left.\operatorname{End}\left(V_{k \omega}^{q}\right)\right)$. Let us realize a braiding of the modules, i.e., construct a $U_{q}(g)$ morphism

$$
\rho: U(g)_{h, q} \rightarrow \operatorname{End}\left(V_{k \omega}^{q}\right)
$$

in the way described above for the algebra $U(\bar{g})=U(g)_{1, q}$. 
Proposition 4 The representation $\rho$ is factorized to a representation of the algebras $A_{h, q}$ with certain $c_{i}(h, q)$. (This means that $\rho\left(V_{\beta}^{q}\right)=0$ if $V_{\beta}^{q} \subset$ $\left.I_{+}^{q} \backslash\left(V_{2 \alpha}^{q} \oplus V_{0}^{q} \oplus V_{+}^{q}\right)\right\}, \rho\left(C_{q}\right)=c_{0}(h, q) \mathrm{id}$, and $\rho\left(V_{+}^{q}\right)=c_{1}(h, q) \rho\left(V^{q}\right)$.)

Proof. It suffices to show that the modules belonging to $I_{+}^{q} \backslash\left(V_{2 \alpha}^{q} \oplus V_{0}^{q} \oplus\right.$ $\left.\left.V_{+}^{q}\right)\right\}$ are not represented in $\operatorname{End}\left(V_{k \omega}^{q}\right)$ for any $k$ and that multiplicity of the modules $V_{0}^{q}$ and $V_{+}^{q}$ in $\operatorname{End}\left(V_{k \omega}^{q}\right)$ is 1 . This can be done by straightforward calculations by means of Young diagram techniques.

Finally, the factors $c_{i}(h, q)$ are defined by these relations. Now if we want to find the relations defining the "quantum orbits" (i.e. algebras corresponding to the case $h=0$ ) we pass to the limits $k \rightarrow \infty$ and $h \rightarrow 0$ in such a way that $c_{0}(h, q)$ has a limit (denoted by $\left.c_{0}(q)\right)$. By virtue of [DS1] $c_{1}(h, q)$ also has a limit (denoted by $c_{1}(q)$ ). These two constants are just the factors that we are looking for.

It is interesting to emphasize that to find the system of equations describing a commutative algebra in the category $U_{q}(g)$-Mod in the framework of this approach, we looking first for the system corresponding to the noncommutative algebra $A_{h, q}(h \neq 0)$.

\section{On "twisted quantum mechanics"}

In this section we discuss the so-called twisted quantum mechanics. Roughly speaking, it is a quantum mechanics in twisted categories. Our aim is to consider the above quantum algebras from this point of view.

It is well known (De Wilde-Lecomte-Fedosov) that any symplectic Poisson structure can be quantized and the result of quantization can be treated as an operator algebra $\operatorname{End}(V)$ in a complex Hilbert space $V$. The space $\operatorname{End}(V)$ is equipped in this case with a trace and a conjugation (involution) possessing the usual properties

$$
\operatorname{tr}(A \circ B)=\operatorname{tr}(B \circ A), \operatorname{tr} A^{*}=\operatorname{tr} A,(A \circ B)^{*}=B^{*} \circ A^{*} .
$$

The quantum observables are identified with Hermitian (self-adjoint) operators in this space and they form a linear space over $\mathbf{R}$ closed with respect to the bracket $i[$,$] .$

If we consider a super-version of quantum mechanics, this bracket must be replaced by its super-analogue. The notions of ordinary trace and conjugation operators must be also replaced by their super-counterparts; this leads, 
in particular, to a modification of the notion of self-adjoint operators. Meanwhile, the observables that play the role of Hamiltonians must be even (since only for an even operator $H$ the equation $d A / d t=i[H, A]$ is consistent) and such an operator is self-adjoint in the usual sense iff it is super-self-adjoint. Moreover, the super-bracket with such an operator becomes the ordinary Lie bracket.

What is a proper analogue of this scheme in a twisted category? If a category under question is symmetric, i.e., the Yang-Baxter twist $S$ is involutive $\left(S^{2}=\mathrm{id}\right)$ in it, the corresponding generalization was suggested in GRZ. In this case "S-analogues" of Lie bracket, trace and conjugation operators introduced there satisfy the following version of the above relations

$$
\operatorname{tr}(A \circ B)=\operatorname{tr} \circ S(A \otimes B), \operatorname{tr} A^{*}=\operatorname{tr} A,(A \circ B)^{*}=\circ(* \otimes *) S(A \otimes B) .
$$

Let us recall a construction of a conjugation operator in this case (assuming $V$ to be finite-dimensional). Let us suppose that we can identify $V$ and $V^{*}$ (this means that there exists a pairing $\langle\rangle:, V^{\otimes 2} \rightarrow k$, which is a morphism of the category). Then $\operatorname{End}(V)$ can be identified with $V^{\otimes 2}$ and the conjugation $*: \operatorname{End}(V) \rightarrow \operatorname{End}(V)$ is just the image of the operator $S: V^{\otimes 2} \rightarrow V^{\otimes 2}$ under this identification. Therefore the conjugation satisfies the following relation $(* \otimes \mathrm{id}) S=S(\mathrm{id} \otimes *)$.

More precisely, we consider the space $g=V$ over the field $k=\mathbf{R}$ and assume all matrix elements of $S$ to be real. This implies that if we introduce an S-Lie bracket in $\operatorname{End}(V)$ by

$$
[A, B]=A \circ B-\circ S(A \otimes B)
$$

its structural constants are real as well.

Let us extend this bracket to the space $V_{\mathbf{C}}=V \otimes \mathbf{C}$ by linearity. Let $*: V_{\mathbf{C}} \rightarrow V_{\mathbf{C}}$ be a conjugation, i.e., an involutive $\left(*^{2}=\mathrm{id}\right)$ operator such that $(\lambda z)^{*}=\bar{\lambda} z^{*}, \lambda \in \mathbf{C}, z \in V_{\mathbf{C}}$. Assume also that the relation $(* \otimes \mathrm{id}) S=$ $S(\mathrm{id} \otimes *)$ and the third relation (6) are satisfied. Then it is easy to see that this conjugation is compatible with the S-Lie bracket in the following sense

$$
*[A, B]=-\left[A^{*}, B^{*}\right] .
$$

It is not so evident what is the proper definition of a conjugation operator, say, in the algebra $\operatorname{End}(V)$, where $V$ is an object of a twisted but nonsymmetric category. Now possessing a q-analogue of the Lie bracket, we can try 
to define the compatibility of such an operator with the twisted structure by means of relation (7). (Let us note that the operator $S$ does not enter explicitly in it and formula (7) is in some sense universal.)

Let us consider a space $V_{\mathbf{C}}$ equipped with a bracket $[]:, V_{\mathbf{C}}^{\otimes 2} \rightarrow V_{\mathbf{C}}$.

Definition 4 We say that a conjugation $*$ is compatible with this bracket if the relation (7) is satisfied.

Proposition 5 The odd elements with respect to this involution (i.e., elements such that $\left.z^{*}=-z\right)$ form a subalgebra, i.e., the element $[a, b]$ is odd if $a$ and $b$ are.

Proof. It is obvious.

Therefore the space of "*-even" operators is closed with respect to the bracket $i[$,$] .$

In [DGR], we have classified all such conjugations for $U_{q}(s l(2))$-case. Let us reproduce the final result here, but first represent the multiplication table for q-Lie bracket in some base $\{u, v, w$,$\} :$

$$
\begin{aligned}
& {[u, u]=0,[u, v]=-q^{2} M u,[u, w]=\left(q+q^{-1}\right)^{-1} M v} \\
& {[v, u]=M u,[v, v]=\left(1-q^{2}\right) M v,[v, w]=-q^{2} M w} \\
& {[w, u]=-\left(q+q^{-1}\right)^{-1} M v,[w, v]=M w,[w, w]=0}
\end{aligned}
$$

Here $M$ is an arbitrary real factor (cf. [G2] for details).

Proposition 6 For a real $q \neq 1$ there exist only two conjugations in the space $V_{\mathbf{C}}$ compatible with q-Lie bracket, namely, that $a^{*}=-\bar{a}$ for any $a \in V_{\mathbf{C}}$ and the following one $u^{*}=u, v^{*}=-v, w^{*}=w$.

Although these conjugations are rather trivial they are, together with quantum traces (their construction in $\operatorname{End}(V)$, where $V$ is an object of a rigid category, is well known) the ingredients of twisted quantum mechanics in the sense of the following informal definition.

Definition 5 We say that an associative algebra is a subject of twisted quantum mechanics if it belongs to a twisted category, it is represented in the space $\operatorname{End}(V)$ equipped with a twisted Lie bracket, a trace and a conjugation as above and the representation map is a morphism in this category. 
Unfortunately, we cannot give a final axiom system of twisted quantum mechanics for a noninvolutive $S$ (though it seems very plausible that the above relations between the operators under consideration are still valid in the category $U_{q}(g)$-Mod if we replace $S$ by $\widetilde{S}$ ). However, we want to point out the principal difference between the twisted version of quantum mechanics and its classical version: the twisted (quantum) trace must occur in calculations of partition functions.

In the above sense the second type quantum algebras represented in some linear spaces in spirit of the paper VS are not subjects of twisted quantum mechanics. Though the algebra $A_{0, q}=\operatorname{Sym}(W)$ itself belongs to the twisted category generated by the space $W$, its modules constructed in VS for $s u(2)$ case do not belong to this category.

As for the second type algebras $A_{h, q}$ with $h \neq 0$, they differ nonessentially from the algebras $A_{0, q}$.

This is not the case for the first type algebra $A_{h, q}$. The representation theory of the algebras $A_{h, q}$ for generic $h$ and that for the case $h=0$ are completely different. From this point of view $h=0$ is a singular point and we disregard it.

Let us note that our first type quantum algebras are subjects of twisted quantum mechanics arising from the quantization of Poisson brackets. However, it is possible to consider similar objects connected to nondeformational solutions of the QYBE (cf. G1, GRZ).

Concluding the paper, we would like to formulate two problems: that of calculating the above factors $c_{i}(h, q)$ (or more generally, giving an exact description of the two parameter deformation of all symmetric orbits in $g^{*}$ for any simple Lie algebra $g$ ) and the problem of generalizing our approach to infinite-dimensional Lie algebras.

\section{References}

[B] R.Börgvad Some homogeneous coordinate rings that are Koszul algebras, alg-geom/951011.

[BG] A.Braverman, D.Gaitsgory Poincaré-Birkhoff-Witt theorem for quadratic algebras of Koszul type, hep-th/9411113. 
[CP] V.Chari, A.Pressley A guide to Quantum Groups, Cambrige University press, 1994

[DG1] J.Donin, D.Gurevich Quantum orbits of R-matrix type, Lett. Math. Phys. 35 (1995), pp. 263-276.

[DG2] J.Donin, D.Gurevich Some Poisson structures associated to DrinfeldJimbo R-matrices and their quantization, Israel Math. Journal 92 (1995), pp.23-32

[DGM] J.Donin, D.Gurevich, S. Majid R-matrix brackets and their quantization Ann. Inst. Henri Poincaré 58 (1993), pp. 235-246

[DGR] J.Donin, D.Gurevich, V.Rubtsov Quantum hyperboloid and braided modules, Proceedings of French-Belgium meeting, 1995, Reims, to appear

[DS1] J.Donin, S.Shnider Quantum symmetric spaces Journal of pure and applied algebra 100 (1995), pp. 103-115

[DS2] J. Donin, S.Shnider Quasi-associativity and flatness criteria for quadratic algebra deformation, Israel Math. J., to appear

[G1] D.Gurevich Algebraic aspects of the quantum Yang-Baxter equation, Leningrad Math.J. 2 (1991), pp.801-828.

[G2] Gurevich D. Braided modules and reflection equations, Publications of Banach Center, Warsaw, to appear

[GP] D.Gurevich, D.Panyushev On Poisson pairs associated to modified R-matrices, Duke Math. J. 73 (1994), pp.249-255.

[GR] D.Gurevich, V.Rubtsov, Quantization of Poisson pencils and generalized Lie algebras, Teor. i Mat. Phys. 103 (1995), pp. 476-488.

[GRZ] D.Gurevich, V.Rubtsov, N.Zobin Quantization of Poisson pairs: $R$ matrix approach, Journ.Geom.and Phys. 9 (1992), pp.25-44

[I] A.Isaev Interrelation between Quantum Groups and Reflection Equation (Braided) Algebras, Lett. Math. Phys. 34 (1995), pp. 333-341. 
[IP] A.Isaev, P.Pyatov Covariant differential complex on quantum linear groups, J. Phys. A: Math. Gen. 28 (1995), pp. 2227-2246.

[KRR] S.Khoroshkin, A.Radul, V.RubtsovFamilies of Poisson structures on Hermitean Symmetric Spases, Comm. Math. Phys. 153 (1993), pp.299-315

[RTF] N.Reshetikhin, L.Takhtadzhyan, L. Faddeev Quantization of Lie groups and Lie algebras, Leningrad Math.J. 1 (1990), pp.S193-226

[S-T] M. Semenov-Tian-Shansky What is classical r-matrix?, Funct. Anal. Appl. 17 (1883), pp.S259-272

[S] E.Sklyanin Some algebraic structures connected with the Yang-Baxter equation Funct. Anal. Appl. 16 (1982), pp.S263-270

[VS] L.Vaksman, Y.Soibelman Algebra of functions on the quantum qroup su(2), Funct. Anal. Appl. 22 (1988), pp.S170-181 